\title{
RELATIONSHIP BETWEEN MAGNETIC PROPERTIES AND MICROSTRUCTURE OF FERRITES DURING SINTERING IN RADIATION AND RADIATION-THERMAL CONDITIONS
}

\author{
Malyshev A.V. ${ }^{1}$, Lysenko E.N. ${ }^{1}$, Sheveleva E.A. ${ }^{1}$, Surzhikova O.A. ${ }^{2}$, Aringazin A.K. $^{3}$ \\ ${ }^{1}$ Tomsk Polytechnic University, Tomsk, Russia, malyshev@tpu.ru \\ ${ }^{2}$ Ceské vysoké ucení technické v Prague, Prague, Czech Republic \\ 3L.N. Gumilev Eurasian National University, Nur-Sultan, Kazakhstan
}

\begin{abstract}
The studies of correlation between magnetic properties and microstructure were conducted on samples of lithium-substituted ferrite, sintered in radiation and radiation-thermal conditions. Radiation-thermal sintering was performed for compacts irradiated with a pulsed electron beam with energy of (1.5-2.0) MeV, beam current per pulse of (0.5-0.9) A, irradiation pulse duration of $500 \mu s$, pulse repetition rate of (5-50) Hz, and compact heating rate of $1000{ }^{\circ} \mathrm{C} / \mathrm{min}$. Sintering in thermal furnaces (T-sintering) was carried out in a preheated chamber electric furnace. The paper shows that magnetic induction does not depend on the ferrite grain size. In this case, the coercive force is inversely proportional to the grain size and depends on the intragranular porosity of ferrite samples. In contrast to thermal sintering, radiation-thermal sintering does not cause capturing of intergranular voids by growing grains and enhances coagulation of intragranular pores.
\end{abstract}

Keywords: ferrites, sintering, magnetic properties, microstructure, intense electron beams, high temperatures.

\section{Introduction}

The use of the effect of ionizing radiation fluxes in production and modification of materials has been observed to grow in recent years [1-5]. Particularly interesting and fundamentally new findings were obtained for a combined effect of powerful radiation fluxes and high temperatures (the so-called radiationthermal effects). The most striking of these findings is the effect of multiple acceleration of synthesis [6-11] and sintering [12-22] of inorganic powder materials. The processes of ferrite material sintering under radiation-thermal effect have been most fully studied for lithium-titanium ferrites and similar structures [2329]. It is obvious that the main functional characteristics of ferrimagnets are their magnetic properties. Attaining a given level of performance properties of ferrites is the ultimate goal of any production technology. Therefore, controlled formation of the main electromagnetic parameters is of high relevance.

However, the processes of magnetization reversal in ferrites are closely related to the features of their microstructure. Therefore, data on the relationship between electromagnetic characteristics and microstructure of ferrites at the stage of their sintering are of interest. This paper presents the results of the study of the kinetic patterns for formation of the hysteresis loop parameters, and patterns of changes in the microstructure of ferrites sintered under thermal and radiation-thermal effects.

\section{Experimental part}

The study uses powders of lithium-titanium ferrite synthesized from a mechanical mixture of oxides and carbonates containing (wt, \%): $\mathrm{Li}_{2} \mathrm{CO}_{3}-11.2 ; \mathrm{TiO}_{2}-18.65 ; \mathrm{ZnO} 7.6 ; \mathrm{MnCO}_{3} 2.74$; others are $\mathrm{Fe}_{2} \mathrm{O}_{3}$. To prepare the compact, $10 \%$ solution of polyvinyl alcohol is added to the synthesized mixture in an amount of 12 wt.\% charge. Compacts in the form of pellets and annular cores with a thickness of $2 \mathrm{~mm}$ are made by cold single-action pressing. Optimum compacting pressure is selected experimentally. It was found that at compacting pressures below $40 \mathrm{MPa}$, press samples exhibit low density, and at compacting pressures of more than $250 \mathrm{MPa}$, samples show cracks and delamination (repressing process).

Thus, the most optimal compacting pressure is in the range of (110-200) MPa. This compacting pressure provides an acceptable density for both green and sintered samples. In the study, the pressing mode used was as follows: $\mathrm{P}=130 \mathrm{MPa}, 1$ min dwelling time; and radiation-thermal (RT) and thermal $(\mathrm{T})$ modes of sintering compacts. In RT-sintering, compacts were exposed to pulsed electron beam with energy of (1.52.0) $\mathrm{MeV}$ using an ILU-6 accelerator. The pulsed beam current was (0.5-0.9) A, the irradiation pulse 
duration was $500 \mu \mathrm{s}$, the pulse repetition rate was $(5-50) \mathrm{Hz}$, and the compact heating rate was $1000^{\circ} \mathrm{C} / \mathrm{min}$. The samples were irradiated in lightweight chamotte box with a wall bottom thickness of $15 \mathrm{~mm}$. The exposed side of the box was covered with a radiation-transparent protector with a mass thickness of 0.1 $\mathrm{g} \cdot \mathrm{cm}^{-1}$. The temperature was measured using a control sample placed in close proximity to the sintered compacts.

T-sintering was performed in a preheated chamber electric furnace, which provided a heating rate comparable to that of radiation heating. The cell design and temperature control technique were similar to those used for RT-sintering. Both sintering modes were performed in air. The magnetic characteristics of the ferrites were measured using the standard technique with an F 5063 ferrometer. The magnetic field was 5 Oe at a frequency of $50 \mathrm{~Hz}$. The degree of porosity and grain sizes were investigated using ground, polished, and etched sections of the sintered samples with an MBI-15U optical microscope. The kinetic dependences of the hysteresis loop parameters $B_{m}, B_{r} / B_{m}$, and $H_{c}$ of ferrite samples were studied after annealing in the temperature range of (1173-1373) $\mathrm{K}[30]$.

\section{Results and discussion}

Figure 1 shows the dependences of the average grain size $d, B_{m}$ and $H_{c}$ on the relative linear shrinkage $\Delta L / L$ on sintering time, since only this mode (in contrast to fast RT-compaction) provides detailed dynamics of the formation of these parameters.

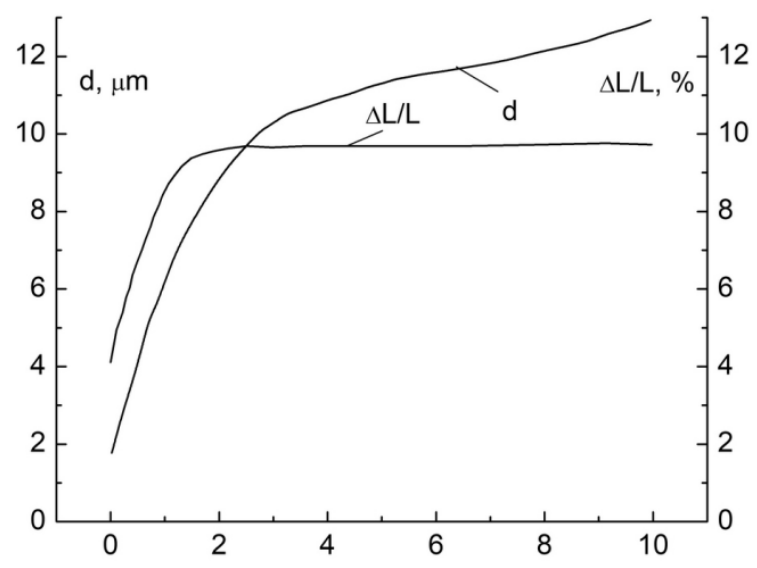

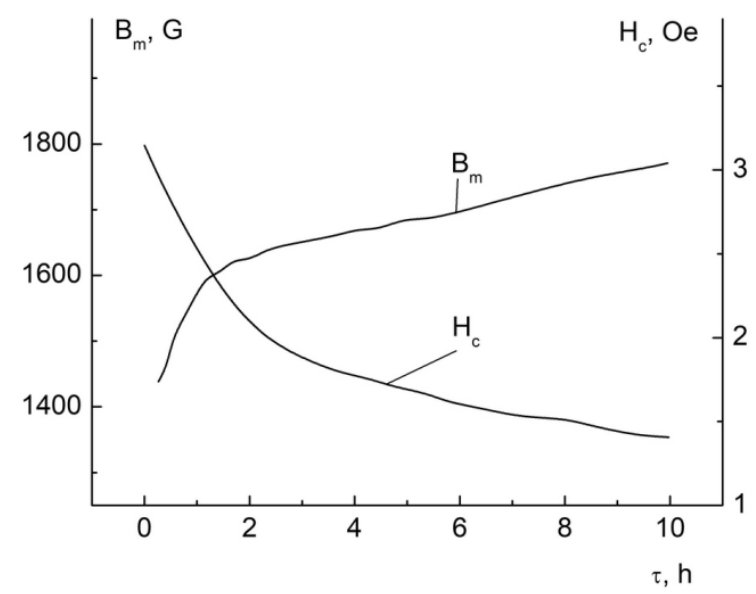

b

Fig.1. Dependencies of average grain size $d$ and $\Delta L / L$ (a), $B_{m}$ and $H_{c}$ (b) of ferrites on time during thermal sintering $\left(\mathrm{T}_{\text {sint }}=1273 \mathrm{~K}\right)$.

This figure shows three stages of sintering. Stage 1 (up to $60 \mathrm{~min}$ ) is accompanied by intense shrinkage of the sample, relatively slow growth of grains, increase in $B_{m}$ and decrease in $H_{c}$. At this stage, interparticle sliding followed by a sharp decrease in intergranular voids (shrinkage), increased concentration of the magnetic phase, and reduced demagnetization effect of the void (increased $B_{m}$ ). Stage 2 (up to 120 min) shows few intergranular voids, shrinkage slows down, $B_{m}$ reaches saturation, and grains continue growing due to formation of numerous grain boundaries. At stage 3 (>120 min), shrinkage is completed, $B_{m}$ is almost constant, grains continue growing (due to collective recrystallization), and $H_{c}$ continues to decrease. This behavior is characteristic of RT-sintering of ferrite compacts. However, the first two phases occur during compact heating and only the last phase can be observed in the isothermal regime.

Thus, the data presented on Figure 1 show the correlation of $H_{c}$ with ferrite porosity and the grain size. No correlation was found between magnetic induction and the grain size. It was previously found that the dependence of $B_{m}$ and $B_{r} / B_{m}$ on porosity is 'apparent', since the maximum induction but not saturation induction was measured at magnetizing field of 5 Oe. The true saturation induction mainly depends on the phase composition and the depth of phase transformations, but it is not related to porosity. Therefore, the microstructure features enable forecasting $H_{c}$ and, conversely, the value of $H_{c}$ indicates the grain size and porosity of the samples. To quantitatively assess this correlation, use the Globus theory [28-30] to calculate the relationship between $H_{c}$ and grain size and compare it with experimental results. 
According to the model proposed by Globus, a ferrite grain contains one boundary. In fields less than $H_{c}$, the edges of the domain boundary remain fixed along the circumference at the grain boundary, and it is observed to bend. The external magnetic field required for irreversible displacement of the 180-degree domain wall is expressed by [31]:

$$
H_{c}=\frac{4 \cdot f}{J_{s} \cdot d},
$$

where $f$ is the force per unit area exerted on the domain interface; $d$ is grain size; $J_{s}$ is saturation magnetization. It is experimentally shown that for various ferrites:

$$
f=0.15 \cdot \sigma_{W},
$$

where $\sigma_{W}$ is the energy of the domain wall per unit area.

The energy of 180-degree domain wall can be expressed by [31]:

$$
\sigma_{W}=\sqrt{\frac{2 A}{K}},
$$

where $A$ is the effective constant of exchange interaction; $\mathrm{K}$ is the crystal anisotropy constant.

The value of the Curie temperature indicates the value of exchange interactions [32]:

$A=\frac{k \Theta}{a}$,

where $k$ is the Boltzmann constant; $a$ is ferrite lattice parameter; $\Theta$ is Curie temperature.

The values of the saturation magnetization and Curie temperature for investigated Li-Ti ferrite are as follows: $J_{s}=142 \mathrm{G} ; \Theta=260^{\circ} \mathrm{C}$. Lattice parameter $a=8.36 \AA$. The value of the anisotropy constant can be taken $K=-5 \cdot 10^{4} \mathrm{erg} / \mathrm{cm}^{3}$.

Appropriate substitutions into equations (1)-(4) yield the theoretical dependence of the $H_{c}$ value on the grain size:

$$
H_{c}=\frac{15.2}{d},
$$

where $H_{c}$ is the coercive force; $d$ is grain size.

It should be emphasized that when inclusions (impurity phases, pores, etc.) occur in the ferrite grain the domain wall in the equilibrium position intersects a certain number of inclusions, since its area and, hence, its energy reduce. In this case, additional energy is spent on magnetization reversal, and $H_{c}$ is expected to increase. This process will involve the inclusions with the size equal to or exceeding the thickness of the domain wall $h[31-32]$ :

$$
h=87 a \sqrt{\frac{\Theta J_{s}}{|K|}},
$$

where $h$ is the domain wall thickness.

Substituting the corresponding data into (5), we obtain $h \approx 1 \mu \mathrm{m}$. Consequently, all inclusions of the size of $r \approx 0.1 \mu \mathrm{m}$ will affect the value of $H_{c}$.

If $H_{c}$ is limited by inclusions in the grain material, the value of $H_{c}$ will be expressed by:

$$
H_{c}=\frac{3 \pi^{1 / 3} \sigma_{W}}{4 J_{s} r}(6 V)^{3 / 2},
$$

where $V=\frac{3 \pi r^{3}}{4 d_{v}^{3}}$ is the volume fraction of inclusions; $r$ is radius of inclusions; $d_{v}$ is distance between inclusions.

Substituting the corresponding values of $\sigma_{W}$ and $J_{s}$ into (7), we obtain:

$$
H_{c}=\frac{27.8}{r}(6 \mathrm{~V})^{3 / 2} \text {, }
$$

The theoretical dependence of $H_{c}$ on the size of inclusions for their various volume fractions is shown in Figure 2. Figure 3 presents the dependencies of $H_{c}$ on the relative volume of inclusions with their sizes being constant.

Figures 2 and 3 show that at high recrystallization rate the capture of intergranular voids into the grain volume increases $H_{c}$. The smaller the radius of these voids, the higher the $H_{c}$ value. The dissociation of captured voids (pores) into smaller ones causes similar effect.

Figure 4 shows the experimental (symbols) and theoretical (solid lines) dependencies of $H_{c}$ on the average ferrite grain size. The experimental and theoretical dependencies for RT-sintering are seen to be in good agreement. This indicates that intergranular voids are not captured into growing grains or that these voids enter the grains in the form of large formations. 


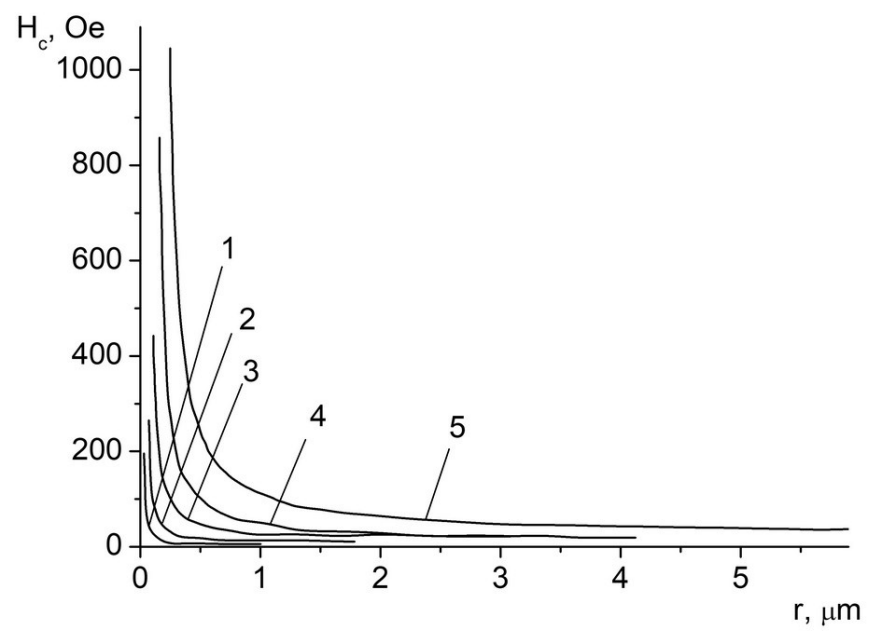

Fig.2. Calculated dependences of $H_{c}$ on the radius of inclusions $r$ at their relative volume $n$ : $1-n=0.05 ; 2-n=0.1 ; 3-n=0.2 ; 4-n=0.3 ; 5-n=0.5$

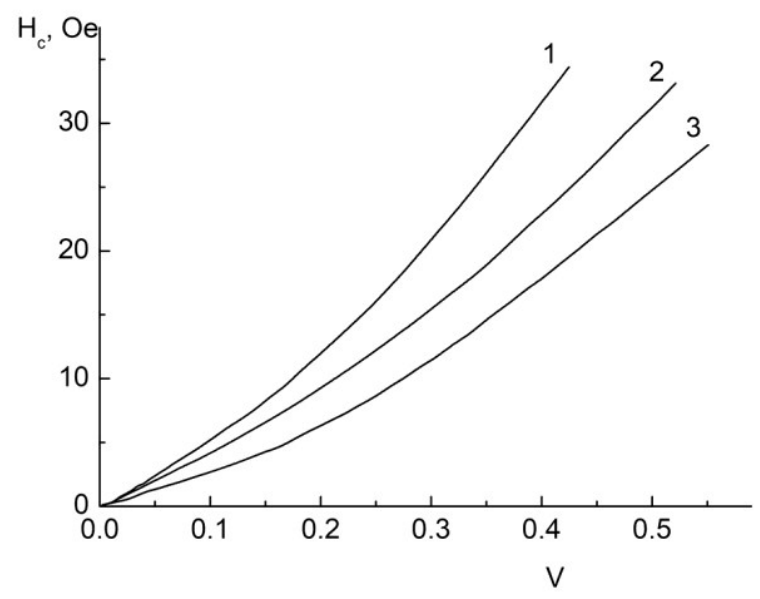

Fig.3. Calculated dependencies of $H_{c}$ on the relative volume of inclusions.

The radius of inclusions $r$ is $3 \mu \mathrm{m}(1) ; 4 \mu \mathrm{m}$ (2); $5 \mu \mathrm{m}$ (3).

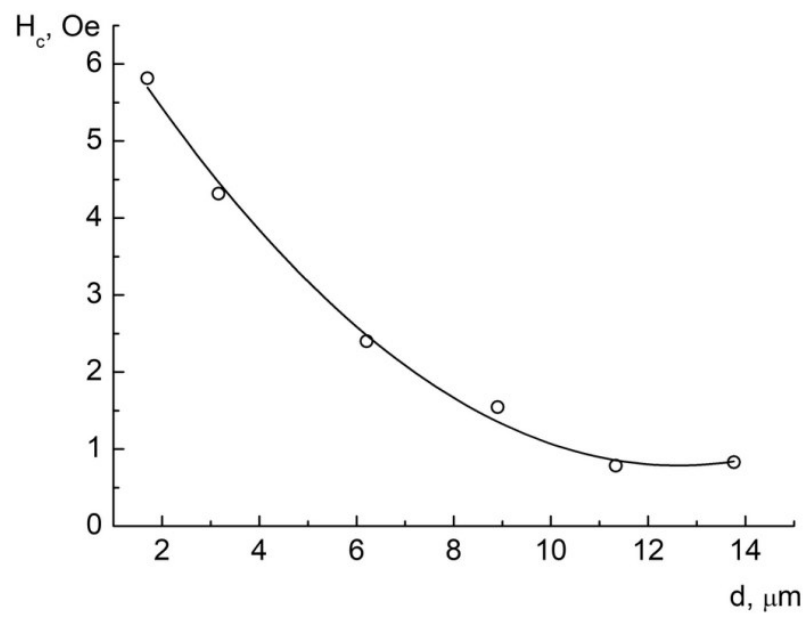

a

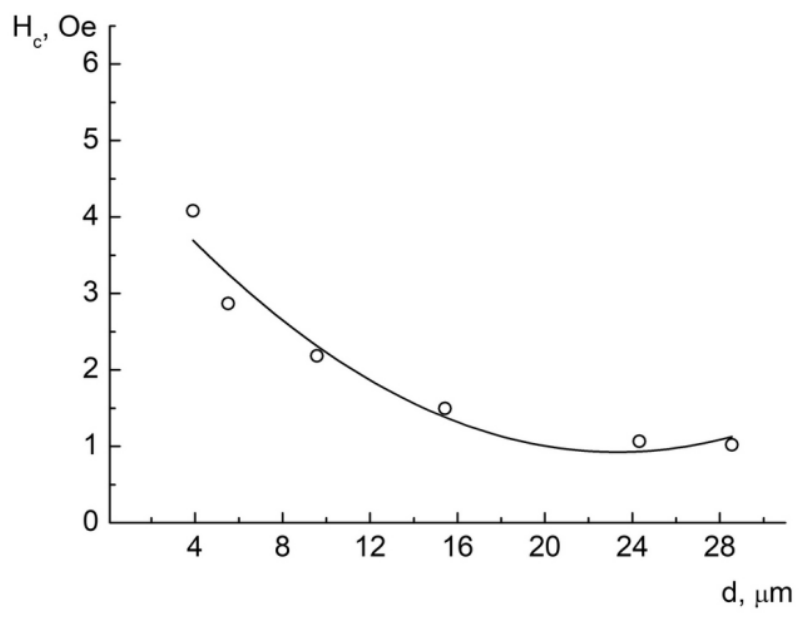

b

Fig.4. Dependence of $H_{c}$ on the average ferrite grain size $d$. Sintering of ferrite samples at $1373 \mathrm{~K}$ in RT mode (a); T-mode (b). Symbols are experimental data; solid lines are calculated dependencies. 
During T-sintering, a coincidence of the calculated and experimental data can be observed only at early sintering stages. As grains grow larger, this coincidence is violated due to intense capturing of small inclusions into the grain volume. The analysis of micrographs of thin sections of ferrite ceramics shows that the capture of intergranular pores during T-sintering is due to growing grains.

\section{Conclusion}

At short sintering time, the grains are predominantly small with a small number of inclusions. The voids are concentrated mainly in the intergranular space. Only individual recrystallized grains contain pores in their volume. Increased sintering time enlarges grains and reduces the volume of intergranular pores. The grains are enriched with inclusions and exhibit insignificant number of pores at the grain boundary. These effects are much weaker in ferrites produced by RT-sintering.

Thus, the data obtained indicate that magnetic induction does not depend on the ferrite grain size. Coercive force is inversely proportional to the grain size and is determined by intragranular porosity. In contrast to $\mathrm{T}$ sintering, RT-sintering does not cause capturing of intergranular voids by growing grains and promotes coagulation of intragranular pores.

It is shown for the first time that using of radiation-thermal sintering in practice makes it possible to obtain lithium-substituted ferrites with a more perfect crystal structure, including without intragranular pores and without entrapment of intergranular voids by growing grains.

\section{Acknowledgments}

\section{The research is funded by the Ministry of Education and Science of the Russian Federation as part of the "Science" Program (project No. FSWW-2020-0014).}

\section{REFERENCES}

1 Groom D.E., Klein S.R. Review of Particle Physics. European Physical Journal C. 1998, Vol.3, pp. 144 - 151.

2 Sagynganova I.K., Markin V.B. The organizations of the tasks implementation in the distributed automatic control systems of heat supply stations. News of the National Academy of Sciences of the Republic of Kazakhstan. 2019, Vol. 1, No. 433, pp. $63-67$.

3 Ershov B.G. Radiation technologies: their possibilities, state, and prospects of application. Herald of the Russian Academy of Sciences. 2013, Vol. 83, No. 5, pp. 437 - 447.

4 Obodovskiy I. Radiation: fundamentals, applications, risks, and safety. Elsevier Inc. 2019, 694 p.

5 Yurov V.M., Baltabekov A.S., Laurinas V.C., Guchenko S.A. Dimensional effects and surface energy of ferroelectric crystals. Eurasian Physical Technical Journal. 2019, Vol. 16, No. 1(31), pp. 18 - 23.

6 Sharma P. Uniyal, Investigating thermal and kinetic parameters of lithium titanate formation by solid-state method, J. Therm. Anal. Calorim. 2017, Vol. 128, pp. $875-882$.

7 Rakshit S.K., Parida S.C., Naik Y.P., Venugopal V. Thermodynamic studies on lithium ferrites, J. Solid State Chem. 2011, Vol. 184, pp. $1186-1194$.

8 Lysenko E.N., Surzhikov A.P., Vlasov V.A., et al. Synthesis of substituted lithium ferrites under the pulsed and continuous electron beam heating. Nuclear Instruments and Methods in Physics Research, Section B: Beam Interactions with Materials and Atoms. 2017, Vol. 392, pp. 1 - 7.

9 Surzhikov A.P., Lysenko E.N., Malyshev A.V., et al. Influence of mechanical activation of initial reagents on synthesis of lithium ferrite. Russian Physics Journal. 2012, Vol. 55, No. 6, pp. 672 - 677.

10 Surzhikov A.P., Pritulov A.M., Lysenko E.N., Sokolovskii A.N., Vlasov V.A., Vasendina E.A. Dependence of lithium-zinc ferrospinel phase composition on the duration of synthesis in an accelerated electron beam. Journal of Thermal Analysis and Calorimetry. 2012, Vol. 110, No. 2, pp. 733-738.

11 Boldyrev V.V., Voronin A.P., Gribkov O.S., Tkachenko E.V., Karagedov G.R., Yakobson B.I., Auslender V.L. Radiation-thermal synthesis. Current achievement and outlook, J. Solid State Ion. 1989, Vol. 36, pp. 1 - 6.

12 Lyakhov N.Z., Boldyrev V.V., Voronin A.P., et al. Electron beam stimulated chemical reaction in solids. Journal of Thermal Analysis. 1995, Vol. 43, pp. 21 - 31. https://doi.org/10.1007/BF02635965.

13 Kostishin V.G., Andreev V.G., Korovushkin V.V., et al. Preparation of 2000NN ferrite ceramics by a complete and a short radiation-enhanced thermal sintering process. Inorganic Materials. 2014, Vol. 50, pp. 317 - 323.

14 Kostishyn V.G., Komlev A.S., Korobeynikov M.V., et al. Effect of a temperature mode of radiation-thermal sintering the structure and magnetic properties of Mn-Zn-ferrites. Journal of Nano-and Electronic Physics. 2015, Vol.7, pp. 04044.

15 Lisitsyn V., Lisitsyna L., Dauletbekova A., et al. Luminescence of the tungsten-activated $\mathrm{MgF}_{2}$ ceramics synthesized under the electron beam. Nuclear Instruments and Methods in Physics Research Section B: Beam Interactions with Materials and Atoms. 2018, Vol. 435, pp. 263 - 267. https://doi.org/10.1016/j.nimb.2017.11.012 
16 Cleland M.R., ParksL.A. Medium and high-energy electron beam radiation processing equipment for commercial applications. Nuclear Instruments and Methods in Physics Research Section B: Beam Interactions with Materials and Atoms. 2003, Vol. 208, pp. 74 - 89. https://doi.org/10.1016/S0168-583X(03)00672-4.

17 Mehnert R. Review of industrial applications of electron accelerators. Nuclear Instruments and Methods in Physics Research Section B: Beam Interactions with Materials and Atoms. 1996, Vol. 113, pp. 81 - 87.

18 Martins M.N., Silva T.F. Electron accelerators: History, applications, and perspectives. Radiation Physics and Chemistry. 2014, Vol. 95, pp. 78 - 85. https://doi.org/10.1016/j.radphyschem.2012.12.008.

19 El-Shobaky G.A., Ibrahim A.A. Solid-solid interactions between ferric oxide and lithium carbonate and the thermal stability of the lithium ferrites produced. Thermochim. Acta. 1987, Vol. 118,pp. 151-158.

20 Salimov R.A., Cherepkov V.G., Golubenko J.I., et al. D.C. high power electron accelerators of ELV-series: status, development, applications. J. Radiation Phys. Chem. 2000, Vol. 57, pp. $661-665$.

21 Cleland M.R., Parks L.A. Medium and high-energy electron beam radiation processing equipment for commercial applications, Nucl. Instr. Meth. B. 2003, Vol. 208, pp. $74-89$.

22 Mehnert R. Review of industrial applications of electron accelerators, Nucl. Instr. Meth. B. 1996, Vol. 113, pp. $81-87$.

23 Neronov V.A., Voronin A.P., Tatarintseva M.I., Melekhova T.E., Auslender V.L. Sintering under a high-power electron beam, J. Less-Common Metals. 1986, Vol. 117, pp. 391 - 394.

24 Surzhikov A.P., Frangulyan T.S., Ghyngazov S.A. A thermoanalysis of phase transformations and linear shrinkage kinetics of ceramics made from ultrafine plasmochemical $\mathrm{ZrO}_{2}(\mathrm{Y})-\mathrm{Al}_{2} \mathrm{O}_{3}$ powders. Journal of Thermal Analysis and Calorimetry. 2014, Vol. 115, No. 2, pp. 1439 - 1445.

25 Surzhikov A.P., Frangulyan T.S., Ghyngazov S.A., et al. Physics of magnetic phenomena: Investigation of electroconductivity of lithium pentaferrite. Rus. Phys. J.2006, Vol. 49, No. 5, pp. $506-510$.

26 Surzhikov A.P., Lysenko E.N., Malyshev A.V., et al. Study of the radio-wave absorbing properties of a lithium-zinc ferrite based composite. Rus. Phys. J. 2014, Vol. 57, No. 5, pp. $621-626$.

27 Surzhikov A.P., Peshev V.V., Pritulov A.M., Gyngazov S.A. Grain-boundary diffusion of oxygen in polycrystalline ferrites. Rus. Phys. J. 1999, Vol. 42, No. 5, pp. $490-495$.

28 Globus A. Some physical consideration about the domain wall sire. Theory of magnetization mechanisms. $J$. Phys. (France). 1977, Vol. 38, No. 4. pp. $1-15$.

29 Globus A. Influence des dimensions des parois la permeability. C. R. Acad. Sci. 1962, Vol. 255, pp. 17091711.

30 Globus A., Guyot M. Wall displacement and bulging in magnetization mechanisms of the hysteresis loop. Phys. Status Solidi B. 1972, Vol. 52, pp. $427-431$.

31 Rankis G., Yurshevich V., Jankovskis J. Modified model of initial magnetic permeability of polycrystalline ferrites. Journal De Physique. IV: JP. 1997, Vol. 7, No. 1, pp. $207-208$.

32 Saulebekov A.O., Venos D., Kambarova Zh.T., et al. Development of energy analyzer of charged particles based on the basis non-uniform electrostatic field. Eurasian Physical Technical Journal. 2019, Vol.16, No.1(31) pp. 24 -29 . 